MATHEMATICS OF COMPUTATION

Volume 68, Number 225, January 1999, Pages 187-199

S 0025-5718(99)00994-1

\title{
ERROR ESTIMATES FOR 3-d NARROW FINITE ELEMENTS
}

\author{
RICARDO G. DURÁN
}

\begin{abstract}
We obtain error estimates for finite element approximations of the lowest degree valid uniformly for a class of three-dimensional narrow elements. First, for the Lagrange interpolation we prove optimal error estimates, both in order and regularity, in $L^{p}$ for $p>2$. For $p=2$ it is known that this result is not true. Applying extrapolation results we obtain an optimal order error estimate for functions sligthly more regular than $H^{2}$. These results are valid both for tetrahedral and rectangular elements. Second, for the case of rectangular elements, we obtain optimal, in order and regularity, error estimates for an average interpolation valid for functions in $W^{1+s, p}$ with $1 \leq p \leq \infty$ and $0 \leq s \leq 1$.
\end{abstract}

\section{INTRODUCTION}

The object of this paper is to analyze the convergence of finite element methods in 3-d when narrow elements are used.

The general standard error analysis (see for example [4]) is based on the so-called "regularity assumption" on the meshes; i.e., the optimal order convergence of finite element approximations, with respect to the meshsize $h$, is obtained provided that the ratio between the outer and inner diameter of the elements remains bounded when $h \rightarrow 0$. However, in 2 -d problems it is known that this condition is not really necessary. Indeed, it can be eliminated in the case of rectangular elements; i.e., the optimal order convergence holds independently of the ratio between edges (see [20] and also [24] where similar results are obtained for the case of isoparametric quadrilaterals), and can be replaced by a "maximum angle condition" (angles bounded away from $\pi$ ) for triangular elements (see [1], [2], [10], [13], [15]).

Consequently, one does not need to avoid very narrow elements which are natural in problems where the solution behaves differently in different directions.

Therefore, a natural question is whether or not these kinds of results can be extended to 3 -d problems. It turns out that the situation is quite different in this case. For the case of tetrahedra, Krízek [16] showed that for the Lagrange interpolation $u^{I}$ of a function $u$, the optimal order convergence is preserved if the angles of the faces and the angles between faces are bounded away from $\pi$, provided $u$ is regular enough. More precisely, he proved that under those assumptions,

$$
\left\|u-u^{I}\right\|_{1, \infty, T} \leq C h|u|_{2, \infty, T},
$$

where $T$ is a tetrahedron, $C$ here and throughout the paper is a generic constant and we have used the standard notation for Sobolev norms and seminorms, \|\|$_{s, p, T}$ and ||$_{s, p, T}$. This angle condition seems to be a natural extension of the 2-d condition.

Received by the editor May 1, 1995 and, in revised form, May 27, 1997.

1991 Mathematics Subject Classification. Primary 65N30. 
However, in many cases $u \notin W^{2, \infty}$, but $u \in W^{2, p}$ for some $p<\infty$. For example, this is the case when $u$ is the solution of an elliptic problem in a nonsmooth domain such as a polyhedron (see [11]). Therefore, one can ask whether an estimate like (1.1) holds with $\infty$ replaced by $p$, with $3 / 2<p \leq \infty$; i.e, $p$ such that the Lagrange interpolation is defined for $u \in W^{2, p}$, precisely,

$$
\left\|u-u^{I}\right\|_{1, p, T} \leq C h|u|_{2, p, T} .
$$

Of particular interest in finite elements is the case $p=2$. For this case $\mathrm{Al}$ Shenk [22] obtained general results which can be applied to many type of degenerate elements in any dimension. His results give optimal order estimates for higher order elements for a wide class of elements. For example, for tetrahedra or 3-d rectangles $T$ and elements of degree $k \geq 2$ he proved that,

$$
\left\|u-u^{I}\right\|_{1,2, T} \leq C h^{k}|u|_{k+1,2, T}
$$

with $C$ a uniform constant for a class of narrow elements. Moreover, Al Shenk gave interesting counterexamples showing that (1.3) does not hold for $k=1$-neither for rectangles nor for tetrahedra. In those examples the constant $C$ blows up when a reference element is compressed in one direction. In particular this shows that (1.3) does not hold for $k=1$ under the Krízek hypothesis.

Then, two natural questions follow:

i) For which values of $p, 2<p \leq \infty$, does (1.2) hold? and,

ii) Which is the regularity required in order to have the optimal order estimate for $p=2$ uniformly valid on narrow elements?

We will show in Section 2 that (1.2) holds for every $p$ such that $2<p \leq \infty$ with a constant $C=C_{p}$ depending on $p$ and, of course, blowing up when $p \rightarrow 2$. In order to answer the second question we give an explicit estimate of the dependence on $p$ for $C_{p}$ which allows us to use known extrapolation results to show that the optimal order is valid for $p=2$ for functions $u$ which are sligthly more regular than $H^{2}$; more precisely, for functions $u \in W_{L^{2}(\log L)^{1+\varepsilon}}^{2}$ (see the definition in Section 2). In other words, we could say that the counterexamples showing that the optimal order in $H^{1}$ does not hold are very "pathological" cases which will not usually arise in practice and therefore the use of narrow elements (in the class of Al Shenk) will not affect the order of convergence in the energy norm.

On the other hand, one could think that the finite element solution of a given problem behaves better than the Lagrange interpolation and that the optimal order is preserved for the finite element approximation even though it does not hold for the interpolation. In view of the results described above this would be of interest for problems where the solution is singular; for example, when the domain is not convex it is usually the case that $u \in H^{1+s}$ for some $0<s<1$, but it is not in $H^{2}$.

In fact, for many problems it is known that,

$$
\left\|u-u_{h}\right\|_{1,2} \leq C \inf _{v_{h} \in V_{h}}\left\|u-v_{h}\right\|_{1,2}
$$

(we do not specify the domain when we are talking of global norms or seminorms), where $V_{h}$ is the finite element space associated with the partition $\mathcal{T}_{h}$ and $C$ depends only on the problem considered. Therefore, to get optimal order convergence it is enough to prove it for some approximation in $V_{h}$. 
In Section 3 we will consider the particular case of 3 -d rectangular elements $R$ and analyze the convergence of an approximation $\Pi u$, where $\Pi$ is an operator of Clement's type, i.e., an average interpolant (see [5], [21]). This kind of interpolant has been introduced and widely used for nonregular functions for which the Lagrange interpolation is not even defined. Our result shows that they can also have better properties than the Lagrange interpolation for more regular functions.

For meshes of 3 -d rectangles we will prove that for $1 \leq p \leq \infty, 0 \leq s \leq 1$ and $u \in W^{1+s, p}$,

$$
\|u-\Pi u\|_{1, p} \leq C h^{s}\|u\|_{1+s, p}
$$

provided that the size of the elements is quasi-uniform in each direction. In other words, the constant $C$ depends on the relation between the element sizes in the $x_{i}$-direction, $i=1,2,3$, but is independent of the relation between the sizes of the edges in different directions. In particular, in view of (1.4), the optimal order of convergence in $H^{1}$ is preserved under these assumptions when $u \in H^{2}$. Moreover, for $s=0$, the quasi-uniformity in each direction can be relaxed by replacing it by a local condition: comparable size in each direction but only between neighbor elements. As we will show, estimates for the case $s=0$ are enough to obtain almost optimal error estimates for finite element approximations of functions in fractional order Sobolev spaces.

\section{ERror estimates FOR the LAGRANGE INTERPOLATION}

In this section we obtain error estimates for the Lagrange interpolation which are uniformly valid for a class of narrow elements. We consider only the case of tetrahedra but analogous results can be proved for rectangular elements in the same way.

First we show that an optimal estimate holds in $W^{1, p}$ for $2<p \leq \infty$. Since the result is not true for $p=2$ (see [22]) the constant in this error estimate blows up when $p \rightarrow 2$. We give an explicit estimate of this constant which allows us to apply extrapolation theory (see for example [18]) to obtain an estimate for $p=2$, which shows that the optimal order is recovered if the interpolated function is sligthly more regular than $H^{2}$.

As in [22] we consider elements $T$ obtained from a reference element $K$ by a compression in each direction followed by an affine transformation given by a matrix $A$ such that, if $\Lambda_{A}$ and $\lambda_{A}$ are its maximum and minimum singular values then,

$$
\frac{\Lambda_{A}}{\lambda_{A}} \leq \gamma
$$

for a given positive constant $\gamma$. We look for error estimates with constants depending only on $\gamma$ and the reference element (in particular, not depending on the relation among the compression factors in each direction).

We derive the error estimates for $T$ being a compression in each direction of the standard reference tetrahedron $K$ with vertices at $(0,0,0), \zeta_{1}=(1,0,0), \zeta_{2}=$ $(0,1,0)$, and $\zeta_{3}=(0,0,1)$. More precisely, $T$ has vertices at $(0,0,0)$ and $h_{i} \zeta_{i}$, $i=1,2,3$ with $h_{i} \leq h$. The constants appearing in the error estimate on $T$ will depend on the reference element and, of course, on $h_{i}$ for $i=1,2,3$ but not on the relations between them. We refer to [22] for the details in the case of a general element obtained from $T$ by an affine transformation satisfying (2.1). 
Given a function $u$ defined on $T$, for $\xi \in K$ and $\xi_{i}=x_{i} / h_{i}$ let

$$
\bar{u}(\xi)=u(x)
$$

and let $u^{I}$ be the linear interpolant of $u$. Then,

$$
\bar{u}^{I}(\xi)=u^{I}(x) .
$$

The error estimate will be a consequence of Lemma 2.1 below. The proof of this lemma is a simple modification of the argument used by Al Shenk [22] and also by Krizek [15] in the two dimensional case. The main idea in [22] and [15] is to introduce a known linear approximation of $u$ and to estimate the difference between it and $u^{I}$. For example, let $B \subset K$ be a ball and $P_{1}(\xi, \eta)$ be the first degree Taylor polynomial of $\bar{u}$ at $\xi \in B$, i.e.,

$$
P_{1}(\xi, \eta)=\bar{u}(\xi)+\nabla \bar{u}(\xi) \cdot(\eta-\xi)
$$

and define,

$$
\bar{u}_{B}(\eta)=\frac{1}{|B|} \int_{B} P_{1}(\xi, \eta) d \xi .
$$

Clearly $\bar{u}_{B} \in \mathcal{P}_{1}$ and it is known that

$$
\left\|\frac{\partial}{\partial \xi_{i}}\left(\bar{u}-\bar{u}_{B}\right)\right\|_{0, p, K} \leq C\left|\frac{\partial \bar{u}}{\partial \xi_{i}}\right|_{1, p, K} ;
$$

indeed, this is the Poincaré inequality applied to the function $\frac{\partial \bar{u}}{\partial \xi_{i}}$. See [7], [8], [9], [12] for different arguments to obtain these types of inequalities and explicit estimates for the constant $C$. In particular, $C$ can be taken independent of $p$ (this follows, for example from the proof given in [8]).

Lemma 2.1. For $2<p \leq \infty$ there exists a positive constant $C_{p}$ such that for $\bar{u} \in W^{2, p}(K)$,

$$
\left\|\frac{\partial}{\partial \xi_{i}}\left(\bar{u}-\bar{u}^{I}\right)\right\|_{0, p, K} \leq C_{p}\left|\frac{\partial \bar{u}}{\partial \xi_{i}}\right|_{1, p, K} .
$$

Proof. In view of (2.2) it is enough to estimate

$$
\left\|\frac{\partial}{\partial \xi_{i}}\left(\bar{u}^{I}-\bar{u}_{B}\right)\right\|_{0, p, K}
$$

Given $v \in \mathcal{P}_{1}(K), \frac{\partial v}{\partial \xi_{i}}$ is a constant and therefore,

$$
\left\|\frac{\partial v}{\partial \xi_{i}}\right\|_{0, p, K}^{p}=\frac{1}{6}\left|\frac{\partial v}{\partial \xi_{i}}\right|^{p}=\frac{1}{6}\left|v\left(\zeta_{i}\right)-v(0)\right|^{p} .
$$

Now, take $v=\bar{u}^{I}-\bar{u}_{B}$ and call $E_{i}$ the edge of $K$ with vertices at 0 and $\zeta_{i}$. We have,

$$
\begin{gathered}
\left|\left(\bar{u}^{I}-\bar{u}_{B}\right)\left(\zeta_{i}\right)-\left(\bar{u}^{I}-\bar{u}_{B}\right)(0)\right|=\left|\left(\bar{u}-\bar{u}_{B}\right)\left(\zeta_{i}\right)-\left(\bar{u}-\bar{u}_{B}\right)(0)\right| \\
=\left|\int_{0}^{1} \frac{\partial}{\partial \xi_{i}}\left(\bar{u}-\bar{u}_{B}\right)\left(t \zeta_{i}\right) d t\right| \leq\left\|\frac{\partial}{\partial \xi_{i}}\left(\bar{u}-\bar{u}_{B}\right)\right\|_{0, p, E_{i}} .
\end{gathered}
$$

The integral of $\frac{\partial}{\partial \xi_{i}}\left(\bar{u}-\bar{u}_{B}\right)$ on the edge is well defined because this function is in $W^{1, p}$ and it is known that in $\mathbb{R}^{3}$, for $p>2$ the functions in $W^{1, p}$ can be restricted to a line and there exists a constant $A_{p}$ such that

$$
\left\|\frac{\partial}{\partial \xi_{i}}\left(\bar{u}-\bar{u}_{B}\right)\right\|_{0, p, E_{i}} \leq A_{p}\left\|\frac{\partial}{\partial \xi_{i}}\left(\bar{u}-\bar{u}_{B}\right)\right\|_{1, p, K} .
$$


Consequently, from (2.3), (2.4) and (2.5) we obtain

$$
\left\|\frac{\partial}{\partial \xi_{i}}\left(\bar{u}^{I}-\bar{u}_{B}\right)\right\|_{0, p, K} \leq A_{p}\left(\left\|\frac{\partial}{\partial \xi_{i}}\left(\bar{u}-\bar{u}_{B}\right)\right\|_{0, p, K}+\left|\frac{\partial \bar{u}}{\partial \xi_{i}}\right|_{1, p, K}\right),
$$

which combined with (2.2) yields the lemma.

Now we can state the error estimate on the element $T$.

Theorem 2.1. For $2<p \leq \infty$, there exists a constant $C_{p}$ depending on $p$ and the reference element $K$ such that for $u \in W^{2, p}$,

$$
\left\|\frac{\partial}{\partial x_{i}}\left(u-u^{I}\right)\right\|_{0, p, T} \leq C_{p}\left(\sum_{j=1}^{3}\left(h_{j}\left|\frac{\partial^{2} u}{\partial x_{j} \partial x_{i}}\right|_{0, p, T}\right)^{p}\right)^{\frac{1}{p}} .
$$

Proof. Changing variables and applying Lemma 2.1 we have

$$
\begin{aligned}
\| \frac{\partial}{\partial x_{i}}(u- & \left.u^{I}\right) \|_{0, p, T}^{p}=\frac{1}{h_{i}^{p}} \int_{K}\left|\frac{\partial}{\partial \xi_{i}}\left(\bar{u}-\bar{u}^{I}\right)\right|^{p} h_{1} h_{2} h_{3} d \xi \\
& \leq \frac{C_{p}^{p}}{h_{i}^{p}} \int_{K} \sum_{j=1}^{3}\left|\frac{\partial^{2} \bar{u}}{\partial \xi_{j} \partial \xi_{i}}\right|^{p} h_{1} h_{2} h_{3} d \xi \\
& =\frac{C_{p}^{p}}{h_{i}^{p}} \int_{T} \sum_{j=1}^{3}\left(h_{j} h_{i}\right)^{p}\left|\frac{\partial^{2} u}{\partial x_{j} \partial x_{i}}\right|^{p} d x
\end{aligned}
$$

and the theorem is proved.

The inequality (2.5) is a particular case of the general classical results about restrictions of functions in Sobolev spaces to lower dimensional surfaces (see for example [17]). In order to obtain a result for $p=2$ applying extrapolation theory, we need an estimate for the constant $A_{p}$ appearing there. Therefore, for this particular case, we will give a short proof which provides an estimate of the constant in terms of $p$. By the standard extension theorem we can work with functions in $W^{1, p}\left(\mathbb{R}^{3}\right)$ having compact support contained in a fixed ball $B(0, M)$, with center at the origin and radius $M$ containing the reference element.

Lemma 2.2. Let $y=\left(y_{1}, y_{2}\right) \in \mathbb{R}^{2}, t \in \mathbb{R}$ and $f(y, t)$ be such that supp $f \subset$ $B(0, M)$ and $f \in W^{1, p}\left(\mathbb{R}^{3}\right)$ with $2<p<3$. There exists a constant $C$ depending on $M$ but independent of $p$ such that,

$$
\int_{\mathbb{R}}|f(0,0, t)|^{p} d t \leq \frac{C}{(p-2)^{\frac{p}{2}}} \int_{\mathbb{R}^{3}}\left|\nabla_{y} f(y, t)\right|^{p} d y d t .
$$

Proof. For almost every $t \in \mathbb{R}$ we have (see for example [23, p. 125])

$$
f(0,0, t)=-\frac{1}{2 \pi} \int_{\mathbb{R}^{2}} \frac{y}{|y|^{2}} \cdot \nabla_{y} f(y, t) d y
$$

therefore, since supp $f \subset B(0, M)$ we obtain,

$$
\begin{aligned}
|f(0,0, t)| & \leq \frac{1}{2 \pi} \int_{|y| \leq \epsilon} \frac{1}{|y|}\left|\nabla_{y} f(y, t)\right| d y+\int_{\epsilon<|y| \leq M} \frac{1}{|y|}\left|\nabla_{y} f(y, t)\right| d y \\
& =\frac{1}{2 \pi}(I+I I),
\end{aligned}
$$

where $\epsilon>0$ will be chosen later. 
To estimate the first term we apply Holder's inequality. Then

$$
I \leq\left(\int_{|y| \leq \epsilon} \frac{1}{|y|^{q}} d y\right)^{\frac{1}{q}}\left(\int_{|y| \leq \epsilon}\left|\nabla_{y} f(y, t)\right|^{p} d y\right)^{\frac{1}{p}}
$$

where $q$ is the conjugate exponent of $p$.

Since $q<2$,

$$
\left(\int_{|y| \leq \epsilon} \frac{1}{|y|^{q}} d y\right)^{\frac{1}{q}}=(2 \pi)^{\frac{1}{q}} \frac{\epsilon^{\frac{2}{q}-1}}{(2-q)^{\frac{1}{q}}}=(2 \pi)^{\frac{1}{q}}(p-1)^{\frac{1}{q}} \frac{\epsilon^{\frac{2}{q}-1}}{(p-2)^{\frac{1}{q}}}
$$

therefore we have

$$
I \leq C \frac{\epsilon^{\frac{2}{q}-1}}{(p-2)^{\frac{1}{q}}}\left(\int_{|y| \leq \epsilon}\left|\nabla_{y} f(y, t)\right|^{p} d y\right)^{\frac{1}{p}}
$$

On the other hand,

$$
I I \leq \frac{1}{\epsilon} \int_{\epsilon<|y| \leq M}\left|\nabla_{y} f(y, t)\right| d y \leq \frac{1}{\epsilon}\left(\pi M^{2}\right)^{\frac{1}{q}}\left(\int_{\epsilon<|y| \leq M}\left|\nabla_{y} f(y, t)\right|^{p} d y\right)^{\frac{1}{p}} .
$$

Therefore, there is a constant $C$ depending on $M$ but not on $p$ such that

$$
|f(0,0, t)|^{p} \leq C\left(\frac{\epsilon^{\frac{2 p}{q}-p}}{(p-2)^{\frac{p}{q}}}+\frac{1}{\epsilon^{p}}\right) \int_{\mathbb{R}^{2}}\left|\nabla_{y} f(y, t)\right|^{p} d y .
$$

Taking $\epsilon=(p-2)^{\frac{1}{2}}$ we have

$$
\frac{\epsilon^{\frac{2 p}{q}-p}}{(p-2)^{\frac{p}{q}}}=\frac{1}{(p-2)^{\frac{p}{2}}}
$$

and therefore, integrating in $t$ we conclude the proof.

From the proof of Lemma 2.1 we know that $C_{p}=C A_{p}$, in particular we obtain the following result.

Corollary 2.1. For $2<p \leq 3$ there exists a constant $C$ depending on the reference element $K$ but independent of $p$ such that for $u \in W^{2, p}$,

$$
\left\|\nabla\left(u-u^{I}\right)\right\|_{0, p, T} \leq \frac{C}{(p-2)^{\frac{1}{2}}} h|u|_{2, p, T} .
$$

As a consequence of Corollary 2.1 we can obtain an optimal order estimate for $p=2$ whenever the function $u$ is sligthly more regular than $H^{2}$. This estimate is obtained applying a result of Milman [19] on extrapolation. In order to state this result and to be precise in what we mean with "sligthly more regular than $H^{2}$ " we recall the definition of the space $L^{2}(\log L(T))^{1+\varepsilon}$ on the element $T$,

$$
L^{2}(\log L(T))^{1+\varepsilon}=\left\{u \in L^{2}: \int_{T} u^{2}(\log (e+|u|))^{1+\varepsilon}<\infty\right\}
$$

as it is known, this is a Banach space with the norm,

$$
\|u\|_{L^{2}(\log L(T))^{1+\varepsilon}}=\inf \left\{\lambda>0: \int_{T}(u / \lambda)^{2}(\log (e+|u| / \lambda))^{1+\varepsilon} \leq 1\right\} .
$$


We will also use the associated Sobolev space $W_{L^{2}(\log L(T))^{1+\varepsilon}}^{2}$ defined as

$$
\begin{array}{r}
W_{L^{2}(\log L(T))^{1+\varepsilon}}^{2}=\left\{u \in L^{2}(\log L(T))^{1+\varepsilon}: D^{\alpha} u \in L^{2}(\log L(T))^{1+\varepsilon},\right. \\
\forall \alpha \text { s.t. }|\alpha| \leq 2\},
\end{array}
$$

which is also a Banach space with the norm,

$$
\|u\|_{W_{L^{2}(\log L(T))^{1+\varepsilon}}^{2}}^{2}=\sum_{|\alpha| \leq 2}\left\|D^{\alpha} u\right\|_{L^{2}(\log L(T))^{1+\varepsilon}}^{2} .
$$

Now we can state the result of Milman [19] which follows from the general theory of extrapolation given in [14] (see also [18]). This result together with Corollary 2.1 implies the desired error estimate,

Lemma 2.3. Let $p_{0}>2$ and $L: W^{2, p}(T) \rightarrow L^{p}(T)$ be a linear operator satisfying

$$
\|L u\|_{0, p, T} \leq \frac{C_{1}}{(p-2)^{\frac{1}{2}}}\|u\|_{2, p, T}
$$

for every $p \in\left(2, p_{0}\right)$. Then, for any $\varepsilon>0$ there exists a constant $C_{2}$ depending only on $C_{1}$ and $\varepsilon$ such that,

$$
\|L u\|_{0,2, T} \leq C_{2}\|u\|_{W_{L^{2}(\log L(T))^{1+\varepsilon}}^{2}} .
$$

Consequently we have the following,

Theorem 2.2. For any $\varepsilon>0$ there exists a constant $C$ depending only on the reference element and $\varepsilon$ such that for $u \in W_{L^{2}(\log L(T))^{1+\varepsilon}}^{2}$,

$$
\left\|\nabla\left(u-u^{I}\right)\right\|_{0,2, T} \leq C h\|u\|_{W_{L^{2}(\log L(T))^{1+\varepsilon}}^{2}} .
$$

Proof. The result is an immediate consequence of Corollary 2.1 and Lemma 2.3.

\section{ERROR ESTIMATES FOR AN AVERAGE INTERPOLANT}

In view of the results in Section 2 a natural question is whether or not optimal order estimates are valid uniformly for narrow elements for other interpolations and more singular functions. In this section we give a positive answer to this question for the particular case of rectangular elements.

Assume that $\mathcal{T}_{h}$ is a partition of 3 -d rectangles with side lengths not greater than $h$ and let $V_{h}$ be the space of continuous piecewise trilinear functions associated with $\mathcal{T}_{h}$.

Given an element $R \in \mathcal{T}_{h}$, denote with $h_{R, i}$ the lengths of the edges of $R$ in the $x_{i}$ directions. Let $\underline{h}_{i}=\min _{R \in \mathcal{T}_{h}} h_{R, i}, h_{i}=\max _{R \in \mathcal{T}_{h}} h_{R, i}$ and, $h=\max _{i=1,2,3} h_{i}$.

We assume that the family of meshes is quasi-uniform in each direction, i.e., there exists a constant $\sigma>0$ such that,

$$
h_{i} / \underline{h}_{i} \leq \sigma
$$

Our error estimates will depend on $\sigma$ but will be independent of the relations between edge sizes on different directions, i.e., they will be valid for narrow elements. The quasi-uniformity in each direction can be relaxed, replacing it by a local condition, if we restrict our estimates to functions in $W^{1, p}$ which, in view of the error estimates for Lagrange interpolation for smooth functions, is enough to obtain almost optimal error estimates for finite element solutions. We will come back to this point later on. 
In order to define the interpolation operator we make a change of variables and transform the mesh into a quasi-uniform reference mesh by a linear transformation. This can be done in view of (3.1). Let

$$
\phi\left(x_{1}, x_{2}, x_{3}\right)=\left(x_{1} / \underline{h}_{1}, x_{2} / \underline{h}_{2}, x_{3} / \underline{h}_{3}\right) .
$$

In this way an element $R$ is transformed into $K$ which have sizes $h_{K, i}$ such that

$$
1 \leq h_{K, i}=h_{R, i} / \underline{h}_{i} \leq \sigma,
$$

and so the mesh $\overline{\mathcal{T}}_{h}=\left\{K=\phi(R): R \in \mathcal{T}_{h}\right\}$ is quasi-uniform.

Given a function $u$ defined on $\Omega$ we define $\bar{u}$ on $\phi(\Omega)$ by

$$
\bar{u}(\xi)=\left(u \circ \phi^{-1}\right)(\xi), \quad \xi \in \phi(\Omega),
$$

and for $\xi=\phi(x)$

$$
\Pi u(x)=\bar{\Pi} \bar{u}(\xi) .
$$

where $\bar{\Pi}$ is defined in the following way.

For a node $\nu=\left(\nu_{1}, \nu_{2}, \nu_{3}\right)$ of $\overline{\mathcal{T}}_{h}$ let $B_{\nu}$ be a ball with center $\nu$ and radius $r$ (fixed from now on and independent of $\nu$ and $h$ ) such that $B_{\nu}$ is contained in the union of elements sharing $\nu$ (clearly we can take any $r<1$ ). If $\nu$ is a boundary node we extend the mesh and the functions $u$ and $\bar{u}$ by standard extension theorems in Sobolev spaces (we do not consider approximations of boundary conditions).

Let $P_{1}(\xi, \eta)$ be as in Section 2 and $\psi$ a $C^{\infty}$ function (also fixed from now on) such that

$$
\text { supp } \psi \subset B(0, r) \text { and, } \int \psi d x=1,
$$

where $B(0, r)$ is the ball with center at the origin and radius $r$.

We define

$$
\bar{u}_{B_{\nu}}(\eta)=\int_{B_{\nu}} P_{1}(\xi, \eta) \psi(\nu-\xi) d \xi
$$

and $\left.\bar{\Pi} \bar{u}\right|_{K}$ as the unique trilinear function such that

$$
\bar{\Pi} \bar{u}(\nu)=\bar{u}_{B_{\nu}}(\nu)
$$

for every vertex $\nu$ of $K$.

Note that, in principle, one could define this interpolation using the definition of $\bar{u}_{B_{\nu}}$ introduced in Section 2. However, we use a regularized average for technical reasons which will become clear below. This interpolation is similar to the one defined by Clement in [5]. We replace the $L^{2}$ projection used in [5] by a regularized average of the Taylor polynomial. This average has been used also in [7], [8] to give a constructive proof of the Bramble-Hilbert lemma. Another difference is that we define the interpolation in a reference patch instead of doing it directly in the patch containing the element considered. Let us also remark that the interpolation $\bar{\Pi}$ can be seen as a particular case of the general interpolation technique given by Scott and Zhang in [21] where they generalize Clement's approach.

For an element $R \in \mathcal{T}_{h}$ we denote by $\widetilde{R}$ the patch formed by $R$ and its neighbor elements and $\widetilde{K}=\phi(\widetilde{R})$.

Our main result is a consequence of the following lemma. 
Lemma 3.1. If (3.1) holds, then for $1 \leq p \leq \infty$ there exist positive constants $C_{3}$ and $C_{4}$, depending only on $r, \psi$ and $\sigma$ such that for $\bar{u} \in W^{2, p}(\widetilde{K})$,

$$
\|\bar{u}-\bar{\Pi} \bar{u}\|_{0, p, K} \leq C_{3}|\bar{u}|_{k, p, \widetilde{K}} \quad \text { for } k=0,1,2,
$$

and

$$
\left\|\frac{\partial}{\partial \xi_{i}}(\bar{u}-\bar{\Pi} \bar{u})\right\|_{0, p, K} \leq C_{4}\left|\frac{\partial \bar{u}}{\partial \xi_{i}}\right|_{k, p, \widetilde{K}} \quad \text { for } k=0,1 .
$$

We postpone the proof of this lemma and show how the error estimates can be derived by changing variables.

Theorem 3.1. If (3.1) holds, then for $1 \leq p \leq \infty$ and $u \in W^{2, p}(\widetilde{R})$,

$$
\begin{gathered}
\|u-\Pi u\|_{0, p, R} \leq C_{3} h^{k}|u|_{k, p, \widetilde{R}} \quad \text { for } k=0,1,2, \\
\left\|\frac{\partial}{\partial x_{i}}(u-\Pi u)\right\|_{0, p, R} \leq C_{4}\left|\frac{\partial u}{\partial x_{i}}\right|_{0, p, \widetilde{R}},
\end{gathered}
$$

and

$$
\left\|\frac{\partial}{\partial x_{i}}(u-\Pi u)\right\|_{0, p, R} \leq C_{4}\left(\sum_{j=1}^{3} h_{j}^{p}\left|\frac{\partial^{2} u}{\partial x_{j} \partial x_{i}}\right|_{0, p, \widetilde{R}}^{p}\right)^{\frac{1}{p}} .
$$

In particular,

$$
\left\|\frac{\partial}{\partial x_{i}}(u-\Pi u)\right\|_{0, p, R} \leq C_{4} h\left|\frac{\partial u}{\partial x_{i}}\right|_{1, p, \widetilde{R}}
$$

where the constants $C_{3}$ and $C_{4}$ are the same as in Lemma 3.1 and so they depend only on $r, \psi$ and $\sigma$ (in particular they are independent of the ratio between edges in different directions).

Proof. We prove only (3.9) since (3.7) and (3.8) can be obtained analogously. Changing variables and applying Lemma 3.1 we have

$$
\begin{aligned}
\| \frac{\partial}{\partial x_{i}} & (u-\Pi u) \|_{0, p, R}^{p}=\frac{1}{\underline{h}_{i}^{p}} \int_{K}\left|\frac{\partial}{\partial \xi_{i}}(\bar{u}-\bar{\Pi} \bar{u})\right|^{p} \underline{h}_{1} \underline{h}_{2} \underline{h}_{3} d \xi \\
& \leq \frac{C_{3}^{p}}{\underline{\underline{h}}_{i}^{p}} \int_{\widetilde{K}} \sum_{j=1}^{3}\left|\frac{\partial^{2} \bar{u}}{\partial \xi_{j} \partial \xi_{i}}\right|^{p} \underline{h}_{1} \underline{h}_{2} \underline{h}_{3} d \xi=C_{3}^{p} \int_{\widetilde{R}} \sum_{j=1}^{3}\left|\frac{\partial^{2} u}{\partial x_{j} \partial x_{i}}\right|^{p} \underline{h}_{j}^{p} d x
\end{aligned}
$$

and the theorem is proved.

An immediate consequence of the theorem is the following

Corollary 3.1. If (3.1) holds then, for $1 \leq p \leq \infty$ and $0 \leq s \leq 1$ there exists a constant $C_{5}$ depending on $C_{3}$ and $C_{4}$ such that for $u \in W^{1+s, p}(\widetilde{R})$,

$$
\|u-\Pi u\|_{k, p, R} \leq C_{5} h^{1+s-k}\|u\|_{1+s, p, \widetilde{R}} \quad \text { for } k=0,1 .
$$

Proof. It follows immediately from Theorem 3.1 and interpolation theory.

Now we prove Lemma 3.1 which is the most technical part of the section. To simplify the notation we consider $i=1$. 
Proof of Lemma 3.1. First we show that

$$
\|\bar{\Pi} \bar{u}\|_{0, p, K} \leq C\|\bar{u}\|_{0, p, \widetilde{K}} .
$$

Let $\nu_{j}, j=1, \ldots, 8$, be the vertices of $K$ and $\chi_{j}$ be the corresponding trilinear Lagrange basis functions. We have

$$
\bar{\Pi} \bar{u}=\sum_{j=1}^{8} \bar{u}_{B_{\nu_{j}}}\left(\nu_{j}\right) \chi_{j}
$$

and then

$$
\|\bar{\Pi} \bar{u}\|_{0, p, K} \leq C \sum_{j=1}^{8}\left\|\bar{u}_{B_{\nu_{j}}}\right\|_{0, \infty, K},
$$

where $C$ is a bound for $\left\|\chi_{j}\right\|_{0, p, K}$ and so, it depends only on $\sigma$. But, from the definition of $\bar{u}_{B_{\nu_{j}}}$ it follows that

$$
\left\|\bar{u}_{B_{\nu_{j}}}\right\|_{0, \infty, K} \leq C\|\bar{u}\|_{0, p, \widetilde{K}},
$$

with $C$ depending on $\psi$ and $\delta=\operatorname{diam}(\widetilde{K})$ and so on $\sigma$ (see [7] for details in the case $p=2$; the argument generalizes straightforwardly for any $p$ ), and therefore (3.11) holds.

Now, since $\bar{\Pi} \bar{u}=\bar{u}$ on $K$ for any $\bar{u} \in \mathcal{P}_{1}(\widetilde{K}),(3.5)$ follows from (3.11) and a standard application of the Bramble-Hilbert lemma. The constant depends on $r$, $\psi$ and $\sigma$. This follows from the direct proofs of that lemma given in [6], [7], [8], [9] or also by a scaling argument.

In order to prove (3.6) we proceed as follows. Let us now call $\nu_{j}$ and $\nu_{j}+e_{1}$, $j=1,2,3,4$, the vertices of $K$ where $e_{1}=\left(h_{K, 1}, 0,0\right)$ with $h_{K, 1}$ being the length of the edges of $K$ in the $x_{1}$ direction. It is known that

$$
\left\|\frac{\partial}{\partial \xi_{1}}\left(\bar{u}-\bar{u}_{B_{\nu_{j}}}\right)\right\|_{0, p, \widetilde{K}} \leq C_{5}\left|\frac{\partial \bar{u}}{\partial \xi_{1}}\right|_{k, p, \widetilde{K}} \quad \text { for } k=0,1,
$$

where $C_{5}$ depends only on $\delta, r$ and $\psi$. Indeed, for $k=0$ this is easily verified using the Young inequality for convolutions, while for $k=1$ this is a particular case of the estimates obtained by Dupont and Scott in [7], [8] (see also [3]); in fact, it is essentially the Poincaré inequality (2.2), the only difference being the fact that we are subtracting a regularized average instead of the average.

Therefore, it is enough to estimate

$$
\left\|\frac{\partial}{\partial \xi_{1}}\left(\bar{u}_{B_{\nu_{1}}}-\bar{\Pi} \bar{u}\right)\right\|_{0, p, K}
$$

and in order to do so we use the following relation which is easy to verify (and is a particular case of a general one stated by $\mathrm{Al}$ Shenk in [22]). For a function $v \in Q_{1}(K)$ (the space of trilinear functions on $K$ ),

$$
\frac{\partial v}{\partial \xi_{1}}=\sum_{j=1}^{4}\left(v\left(\nu_{j}+e_{1}\right)-v\left(\nu_{j}\right)\right) \frac{\partial \chi_{j}}{\partial \xi_{1}},
$$

where $\chi_{j}$ are now the basis functions corresponding to the node $\nu_{j}+e_{1}$. Therefore,

$$
\left\|\frac{\partial v}{\partial \xi_{1}}\right\|_{0, p, K}^{p} \leq C \sum_{j=1}^{4}\left|v\left(\nu_{j}+e_{1}\right)-v\left(\nu_{j}\right)\right|^{p},
$$


where $C$ depends only on $\sigma$. In fact, it depends on the norms $\left\|\frac{\partial \chi_{j}}{\partial \xi_{1}}\right\|_{0, p, K}$, which in view of (3.2) are bounded in terms of $\sigma$.

Consequently, taking $v=\bar{u}_{B_{\nu_{1}}}-\bar{\Pi} \bar{u}$ the lemma will be proved if we show that for $k=0,1$ and $j=1,2,3,4$,

$$
\left|\left(\bar{u}_{B_{\nu_{1}}}-\bar{\Pi} \bar{u}\right)\left(\nu_{j}+e_{1}\right)-\left(\bar{u}_{B_{\nu_{1}}}-\bar{\Pi} \bar{u}\right)\left(\nu_{j}\right)\right| \leq C\left|\frac{\partial \bar{u}}{\partial \xi_{1}}\right|_{k, p, \widetilde{K}}
$$

with $C$ depending only on $\sigma, \psi$ and $r$.

In order to simplify the notation we call

$$
B_{0}=B_{\nu_{1}}, B_{1}=B_{\nu_{j}}, B_{2}=B_{\nu_{j}+e_{1}}, \eta_{1}=\nu_{j}, \eta_{2}=\nu_{j}+e_{1} .
$$

Now

$$
\begin{aligned}
& \left|\left(\bar{u}_{B_{0}}-\bar{\Pi} \bar{u}\right)\left(\eta_{2}\right)-\left(\bar{u}_{B_{0}}-\bar{\Pi} \bar{u}\right)\left(\eta_{1}\right)\right| \\
& \quad=\left|\bar{u}_{B_{0}}\left(\eta_{2}\right)-\bar{u}_{B_{2}}\left(\eta_{2}\right)-\bar{u}_{B_{0}}\left(\eta_{1}\right)+\bar{u}_{B_{1}}\left(\eta_{1}\right)\right| \\
& \quad \leq\left|\bar{u}_{B_{0}}\left(\eta_{2}\right)-\bar{u}_{B_{1}}\left(\eta_{2}\right)-\bar{u}_{B_{0}}\left(\eta_{1}\right)+\bar{u}_{B_{1}}\left(\eta_{1}\right)\right|+\left|\bar{u}_{B_{2}}\left(\eta_{2}\right)-\bar{u}_{B_{1}}\left(\eta_{2}\right)\right| \\
& \quad=I+I I .
\end{aligned}
$$

Since $\frac{\partial}{\partial \xi_{1}}\left(\bar{u}_{B_{0}}-\bar{u}_{B_{1}}\right)$ is a constant we have

$$
\begin{aligned}
I & =\left|\int_{0}^{1} \frac{\partial}{\partial \xi_{1}}\left(\bar{u}_{B_{0}}-\bar{u}_{B_{1}}\right)\left(\eta_{1}+t e_{1}\right) h_{K, 1} d t\right| \\
& \leq \sigma|K|^{-1 / p}\left\|\frac{\partial}{\partial \xi_{1}}\left(\bar{u}_{B_{0}}-\bar{u}_{B_{1}}\right)\right\|_{0, p, K} \leq C_{5} \sigma\left|\frac{\partial \bar{u}}{\partial \xi_{1}}\right|_{k, p, \widetilde{K}}
\end{aligned}
$$

for $k=0,1$ where we have used (3.12) and the fact that $|K| \geq 1$ for the last inequality.

For the second term we change variables to obtain

$$
\begin{aligned}
I I & =\left|\bar{u}_{B_{2}}\left(\eta_{2}\right)-\bar{u}_{B_{1}}\left(\eta_{2}\right)\right| \\
& =\left|\int_{B_{2}} P_{1}\left(\xi, \eta_{2}\right) \psi\left(\eta_{2}-\xi\right) d \xi-\int_{B_{1}} P_{1}\left(\xi, \eta_{2}\right) \psi\left(\eta_{1}-\xi\right) d \xi\right| \\
& =\left|\int_{B_{1}}\left[P_{1}\left(\xi+e_{1}, \eta_{2}\right)-P_{1}\left(\xi, \eta_{2}\right)\right] \psi\left(\eta_{1}-\xi\right) d \xi\right| .
\end{aligned}
$$

If we set $F(t)=P_{1}\left(\xi+t e_{1}, \eta_{2}\right)$, then

and therefore,

$$
F^{\prime}(t)=\frac{\partial}{\partial \xi_{1}} \nabla \bar{u}\left(\xi+t e_{1}\right) h_{K, 1} \cdot\left(\eta_{2}-\left(\xi+t e_{1}\right)\right)
$$

$$
I I=\left|\int_{B_{1}} \int_{0}^{1} \frac{\partial}{\partial \xi_{1}} \nabla \bar{u}\left(\xi+t e_{1}\right) h_{K, 1} \cdot\left(\eta_{2}-\left(\xi+t e_{1}\right)\right) \psi\left(\eta_{1}-\xi\right) d t d \xi\right| .
$$

Now, for $t \in[0,1]$ we have

$$
\begin{aligned}
& \left|\int_{B_{1}} \frac{\partial}{\partial \xi_{1}} \nabla \bar{u}\left(\xi+t e_{1}\right) h_{K, 1} \cdot\left(\eta_{2}-\left(\xi+t e_{1}\right)\right) \psi\left(\eta_{1}-\xi\right) d \xi\right| \\
& \quad \leq C \sigma \delta \int_{B_{1}}\left|\frac{\partial}{\partial \xi_{1}} \nabla \bar{u}\left(\xi+t e_{1}\right)\right| d \xi \leq C \sigma \delta\left|\frac{\partial \bar{u}}{\partial \xi_{1}}\right|_{1, p, \widetilde{K}}
\end{aligned}
$$

where $C$ depends on $\psi$. This estimate together with (3.14) and (3.15) proves (3.13) for $k=1$. 
On the other hand, integration by parts yields

$$
\begin{aligned}
& \mid \int_{B_{1}} \frac{\partial}{\partial \xi_{1}} \nabla \bar{u}\left(\xi+t e_{1}\right) h_{K, 1} \cdot\left(\eta_{2}-\left(\xi+t e_{1}\right)\right) \psi\left(\eta_{1}-\xi\right) d \xi \mid \\
& \quad=\left|-\int_{B_{1}} \frac{\partial \bar{u}}{\partial \xi_{1}}\left(\xi+t e_{1}\right) h_{K, 1} \operatorname{div}\left[\left(\eta_{2}-\left(\xi+t e_{1}\right)\right) \psi\left(\eta_{1}-\xi\right)\right] d \xi\right| \\
& \quad \leq C \sigma\left|\frac{\partial \bar{u}}{\partial \xi_{1}}\right|_{0, p, \widetilde{K}}
\end{aligned}
$$

with $C$ depending on $\delta$ and $\psi$. Using again (3.14) and (3.15) we have proved the lemma.

\section{EXTENSIONS AND FINAL REMARKS}

We have obtained error estimates for finite element approximations which are valid uniformly for a class of narrow elements. Clearly, the results of Section 2 can also be proved for the case of rectangular elements with simple modifications.

On the other hand, the estimates (3.7) for $k=0,1$ and (3.8) hold under weaker assumptions. Indeed, the hypothesis (3.1) on quasi-uniformity in each direction can be replaced by a local condition: comparable edge size in each direction but only for neighbor elements. In fact, we can replace the linear transformation $\phi$ by a piecewise linear one which transforms the mesh $\mathcal{T}_{h}$ into a uniform reference mesh of unit cubes. Then, we define the interpolation operator as in (3.3) but using this new transformation, and it is easy to see that, under this local condition, the arguments of the proof can be repeated to obtain those estimates for functions $u \in W^{1, p}$. For (3.7) with $k=2$ and (3.9) this cannot be done because this new transformation does not preserve $W^{2, p}$ regularity. However, the estimates for $W^{1, p}$ functions are the most important because for $u$ more regular we can use the Lagrange interpolation to obtain error estimates for the finite element approximation on meshes with narrow elements. In this way, by using the results of Section 2 combined with (3.7) and (3.8) for $u \in W^{1, p}$ we can derive almost optimal error estimates for finite element approximations when $u \in W^{s, p}, 1 \leq s \leq 2$, and $p>2$ from Banach space interpolation theory. Indeed, if (1.4) holds we obtain from (2.7), (3.7) and (3.8) that for $p>2$

$$
\left\|u-u_{h}\right\|_{1,2} \leq C h\|u\|_{2, p}
$$

with $C$ depending on $p$ as in $(2.7)$ and

$$
\left\|u-u_{h}\right\|_{1,2} \leq C\|u\|_{1,2},
$$

and, therefore, by interpolation we obtain

$$
\left\|u-u_{h}\right\|_{1,2} \leq C h^{s}\|u\|_{1+s, p},
$$

which is optimal in order and almost optimal in regularity since $p>2$ (and we could use extrapolation as in Section 2 to obtain an estimate analogous to that in Theorem 2.2 for the finite element aproximation in fractional order Sobolev norms).

The extension of the results obtained in Section 3 for the case of tetrahedra would be interesting. It is not straightforward and is the object of our current research. Results for degenerate tetrahedra and singular functions would be of 
interest since, for problems with singular solutions, local refinement of the meshes is needed and, as far as the author knows, the known procedures do not avoid the use of narrow elements. This is different than in the 2 -d case where it is known that the refinement can be done without using small angles.

\section{REFERENCES}

[1] I. Babuska and A.K. Aziz, On the angle condition in the finite element method, SIAM J. Numer. Anal. 13 (1976), 214-226. MR 56:13700

[2] R. E. Barnhill and J.A. Gregory, Interpolation remainder theory from Taylor expansions on triangles, Numer. Math. 25 (1976), 401-408. MR 56:7253

[3] S.C. Brenner and L.R. Scott, The Mathematical Theory of Finite Element Methods, Texts in Applied Mathematics, vol.15, Springer-Verlag, 1994. MR 95f:65001

[4] P.G. Ciarlet, The Finite Element Method for Elliptic Problems, North Holland, 1978. MR 58:25001

[5] P. Clement, Approximation by finite element functions using local regularization, RAIRO Anal. Numér. 9 (1975), 77-84. MR 53:4569

[6] L.T. Dechevski and E. Quak, On the Bramble-Hilbert lemma, Numer. Funct. Anal. and Optimiz. 11 (1990), 485-495. MR 91k:46027

[7] T. Dupont and R. Scott, Constructive polynomial approximation in Sobolev spaces, Recent Advances in Numerical Analysis (C.de Boor and G. Golub, eds.), Academic Press, 1980. MR 80j: 41055

[8] , Polynomial approximation of functions in Sobolev spaces, Math. Comp. 34 (1980), 441-463. MR 81h:65014

[9] R.G. Durán, On polynomial approximation in Sobolev spaces, SIAM J. Numer. Anal. 20 (1983), 985-988. MR 85e:42010

[10] J.A. Gregory, Error bounds for linear interpolation on triangles, The Mathematics of Finite Elements and Applications II, Academic Press, 1976, pp. 163-170. MR 56:16995

[11] P. Grisvard, Elliptic Problems in Nonsmooth Domains, Pitman, 1985. MR 86m:35044

[12] S.M. Hudson, Polynomial approximation in Sobolev spaces, Indiana University Math. J. 39 (1990), 199-228. MR 91h:41007

[13] P. Jamet, Estimations d'erreur pour des éléments finis droits presque dégénérés, RAIRO Anal. Numér 10 (1976), 46-61. MR 56:13521

[14] B. Jawerth and M. Milman, Extrapolation theory with applications, Memoirs Amer. Math. Soc. 440 (1991). MR 91i:46092

[15] M. Krízek, On semiregular families of triangulations and linear interpolation, Applications of Math. 36 (1991), 223-232. MR 92e:65010

[16] _ On the maximum angle condition for linear tetrahedral elements, SIAM J. Numer. Anal. 29 (1992), 513-520. MR 92k:65165

[17] Ladyzhenskaya, Solonnikov and Uraltceva, Linear and Quasilinear Parabolic Differential Equations, Transl. of Math. Mon., vol. 23, AMS, 1968.

[18] M. Milman, Extrapolation and Optimal Decompositions, Lecture Notes in Mathematics, vol. 1580, Springer-Verlag, Berlin, 1994. MR 96a:46133

[19] , Personal communication.

[20] M.H. Schultz, Spline Analysis, Prentice Hall, 1973. MR 50:15270

[21] L.R. Scott and S. Zhang, Finite element interpolation of non-smooth functions satisfying boundary conditions, Math. Comp. 54 (1990), 483-493. MR 90j:65021

[22] N. Al Shenk, Uniform error estimates for certain narrow Lagrange finite elements, Math. Comp. 63 (1994), 105-119. MR 94i:65119

[23] E. Stein, Singular Integrals and Differentiability Properties of Functions, Princeton University Press, 1970. MR 44:7280

[24] A. Zenísek and M. Vanmaele, The interpolation theorem for narrow quadrilateral isoparametric finite elements, Numer. Math. 72 (1995), 123-141. MR 97i:65157

Departamento de Matemática, Facultad de Ciencias Exactas, Universidad de Buenos Aires, (1428) Buenos Aires, Argentina 